\title{
latrogene Schäden - Fehlervermeidung in Orthopädie und Unfallchirurgie
}

\author{
latrogenic Injuries - Avoiding Errors in Orthopaedics and Traumatology
}

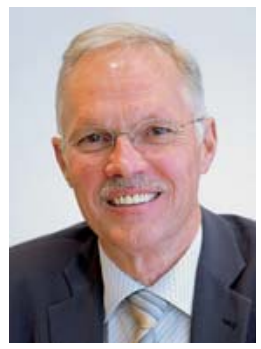

F. U. Niethard

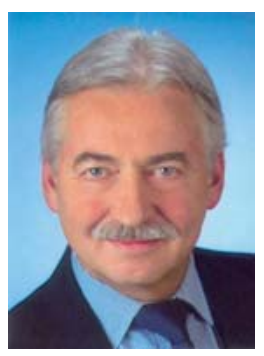

K. Weise

Bibliografie

DOI 10.1055/s-2008-1038480 Z Orthop Unfall 2008; 146: 173-174 @ Georg Thieme Verlag KG Stuttgart • New York . ISSN 1864-6697

\section{Korrespondenzadresse} Prof. Dr. med. Fritz U. Niethard Orthopädische Klinik Universitätsklinik der RWTH Aachen Pauwelsstraße 30 52074 Aachen Tel.: 0241/808-94 10 Fax: 0241/808-2453 funiethard@ orthopaedie-aachen.de

Prof. Dr. med. Kuno Weise BG-Unfallklinik

Schnarrenbergstraße 95 72076 Tübingen

Tel.: $07071 / 606-1001$

Fax: 07071/606-1002 weise@bgu-tuebingen.de
.... wegen eines vereiterten Zahnes erhält ein Patient eine Penicillin-Spritze, gegen die er aber allergisch ist und einen Schock erleidet. Dieser wird mit hochdosierten Kortikosteroiden behandelt, was zum blutenden Magenulcus führt, das wiederum - weil unstillbar - chirurgisch angegangen werden muss. Im Zusammenhang mit der Magenoperation erhält der Patient mehrere Blutkonserven mit den Folgen einer Hepatitis. Letztlich verstirbt der Patient im Coma hepaticum...

Dieses fiktive Horrorszenario wird bereits Anfang der 70er-Jahre in einem Kongress über „Iatrogenic diseases“ beschrieben, um auf die Gefährlichkeit der modernen Medizin und die sog. „side effects“ hinzuweisen. Die erste in Medline erfasste Literatur zu diesem Thema reicht sogar bis Anfang 1950 zurück und das „primum nil nocere“ als Wahlspruch aus der hippokratischen Tradition ist zentrales Gedankengut der Medizin bis heute.

Die Ende Februar 2008 vom „Aktionsbündnis Patientensicherheit" [1] vorgestellte Publikation „Aus Fehlern lernen“ scheint allerdings zu vermitteln, dass sich die Ärzte erst jetzt ihres Tun und Handelns bewusst sind und die Patientensicherheit als Aufgabe erkannt haben. In dieser Broschüre bekennen sich Ärztinnen und Ärzte, Mitarbeiterinnen und Mitarbeiter von pflegerischen und therapeutischen Berufen zu eigenen Fehlern und Beinah-Fehlern und haben damit eine Flut an Reaktionen in Medien ausgelöst; denn „Fehler, noch dazu ärztliche, sind für Journalisten ein dankbares Thema“. Kommt es zu einer Seitenverwechslung in der Chirurgie oder wurde einem Patienten ein falsches Medikament verabreicht, stürzen sich diese darauf. Getreu dem Motto „Jeder Fehler erscheint unglaublich dumm, wenn andere ihn begehen“ [2].

Von zahlreichen Vertretern der Gesundheitsverbände wurde die Initiative begrüßt, sei sie doch ein Einstieg in eine neue Fehlerkultur. Dieser Einstieg war - wohlgemeint - vom Präsidenten der Deutschen Gesellschaft für Chirurgie schon 2005 anlässlich des 122. Chirurgen-Kongresses bewusst gewählt worden. Heraus kam eine „ungewollte Verunsicherung“ [3]. Denn in den Medien hieß es, dass mehr Tote durch Ärztepfusch als im Straßenverkehr zu beklagen seien. Und so schlug denn die beabsichtigte Offenheit im Umgang mit ärztlichen Fehlern in das Gegenteil um: eine Angstkampagne ohne jeglichen Ansatz für einen differenzierten Umgang mit dem Thema. Ein Thema, das vorrangig die chirurgischen Fächer zu betreffen scheint. Inwieweit sind denn nun auch Orthopädie und Unfallchirurgie involviert?

Nach einer Statistik über die Verfahren in der norddeutschen Schlichtungsstelle sind Orthopädie und Unfallchirurgie gemeinsam sogar am häufigsten betroffen ( $\bullet$ Tab. 1 ): Ungefähr ein Drittel der in der norddeutschen Schlichtungsstelle 2000 - 2003 anhängigen Verfahren betrifft Orthopädie und Unfallchirurgie. Die häufigsten Diagnosen sind: Extremitätenfrakturen, Cox- und Gonarthrose, Bandscheiben- und Kniegelenkschäden [4]. Ist hier etwa doch eine bedrohliche Entwicklung in der Orthopädie und Unfallchirurgie im Gange, ohne dass sie von den Fachgesellschaften bemerkt worden wäre?

In der Statistik von Scheppokat und Neu [4] sind ca. $30 \%$ der Patientenschäden fehlerverursacht und die Patientenansprüche somit begründet. Als wesentliche Ursachen von Patientenschäden werden die hochkomplexen operativen Eingriffe (und damit die Kompetenz des Operateurs) sowie Kommunikationsdefizite aufgeführt. Damit sind die beiden Komplexe „Aus-, Weiter- und Fortbildung“ sowie „Struktur- und Prozessqualität“ angesprochen, um die sich die Fachgesellschaften seit jeher bemühen.

Es ist nicht zum ersten Mal, dass sich auch die Zeitschrift für Orthopädie und Unfallchirurgie mit der Qualitätssicherung im Fach beschäftigt [5-7]. In diesem Heft nun wird in der Arbeit „Risikomanagement zur Fehlervermeidung im Krankenhaus“ nur eine der zahlreichen Einflussgrößen auf die Ergebnisqualität näher beleuchtet. Es geht um die Kommunikation unter Ärzten, die umso mehr geschult werden muss, je häufiger und je intensiver sie beansprucht wird [8] Die ungebrochene Ökonomisierung des Gesundheitssystems, die sich daraus ergebende Personalnot [9], neue Versorgungsstrukturen (z.B. fachübergreifende Dienste), Arbeitszeitgesetze und überbordende Demokratie beeinträchtigen die Patientenversorgung nicht erst heute [10]. Auf die sich daraus ergebenden Konsequenzen haben die Fachgesellschaften DGOOC und DGU wiederholt hingewiesen. Die Autoren der o.g. Arbeit betonen daher zu Recht, dass die organisatorischen Rahmenbedingungen, unter denen Ärzte und Pflegekräfte in Akut-Kliniken arbeiten, bereits durch hohe Belastungsspitzen besonders fehleranfällig sind. In solchen Situationen auftre- 
Tab. 1 Verfahren in der norddeutschen Schlichtungsstelle 2000 - 2003, Fachgebiete der Ärzte/Krankenhäuser [4]

\begin{tabular}{lcc} 
Chirurgie ohne Unfallchirurgie & 2706 & \\
Chirurgie/Teilgebiete & 4876 & \\
$\begin{array}{l}\text { - davon Unfallchirurgie } \\
\text { Orthopädie }\end{array}$ & 1456 & \\
\hline Orthopädie und Unfallchirurgie & & 3626 \\
Frauenheilkunde, Geburtshilfe & 1557 & \\
Innere Medizin/Teilgebiete & 1011 &
\end{tabular}

tende Kommunikationsprobleme und Missverständnisse gehören zu den häufigsten Fehlerursachen in der Medizin. Es ist daher naheliegend, sich Vorbilder z. B. aus der Luftfahrt zu beschaffen, die Vorgaben für eine strukturierte Kommunikation bieten. Die Luftfahrt hat ja auch bei der Einführung des sog. „critical incident reporting system" (CIRS) Pate gestanden, das in der Schweiz und nun auch in Deutschland Licht in die vermeintlich große Dunkelziffer von „Beinahe-Unfällen“ oder Unfällen bringen soll [11]. Ein derartiges System ist nachhaltig zu unterstützen, wenn es denn nicht von der Sensationsgier der Medien instrumentalisiert und ausgehöhlt wird. Wenn denn schon zunehmend häufiger der Vergleich zwischen Chirurg und Pilot hergestellt wird: ein „Aktionsbündnis Fluggastsicherheit“ und ein öffentliches Bekenntnis von Pilotinnen und Piloten zu ihren Fehlern und Beinahe-Fehlern ist bisher nicht bekannt. Dass die Ergebnisqualität bei der Behandlung von Patienten nicht erst durch ein „critical incident reporting system“ überprüft werden muss, ist den Gesellschaften DGOOC und DGU allgegenwärtig. Die Orthopädie ist originär präventiv und damit auf die Vermeidung von Schäden ausgerichtet. Qualitätssichernde Maßnahmen durch die Etablierung von Mindestmengen oder auch durch die Einrichtung eines Endoprothesenregisters werden seit Langem gefordert. Die Unfallchirurgie weiß, dass der Wettlauf der lebensbedrohlichen Situation eines Schwerverletzten nur durch eine entsprechende Struktur- und Prozessqualität gewonnen werden kann. Entsprechend hoch muss die Arbeit von Kommissionen, berufsständischen Ausschüssen und Anderen gewichtet werden, die sich mit der Erstellung von Leitlinien bis hin zur Abfassung des „Weißbuches Schwerverletztenversorgung“ [12] beschäftigt haben. Zur Verbesserung der Versorgung von Verletzten vom Unfallort bis in die klinische Versorgung wurde nun zusätzlich das Programm „Safe : trac“ auf den Weg gebracht.
Nicht zuletzt aber ist der Einsatz der Fachgesellschaften für Aus-, Weiter- und Fortbildung zu würdigen, denn Beinahe-Fehler können vom kompetenten Arzt antizipiert und von vornherein vermieden werden. So gesehen ist die Fehlerbekenntnis von Ärztinnen und Ärzten in der Broschüre des „Aktionsbündnisses Patientensicherheit“ eine deutliche Warnung gegenüber den Empfehlungen des Sachverständigenrates zur Begutachtung der Entwicklung im Gesundheitswesen. Dieser hatte ja die „Arztzentriertheit" des Gesundheitswesens kritisiert und dazu aufgerufen, anderen ärztlichen Gesundheitsberufen mehr medizinische Verantwortung zu übertragen. Dass die unkritische Delegation ärztlicher Leistungen auf nicht ärztliche Berufe Kompetenz abbaut und neue Schnittstellen entstehen lässt und damit auch Risiken heraufbeschwört, ist nach Meinung des Fachanwaltes für Medizinrecht Dr. jur. Albrecht Wienke schon jetzt vorauszusehen [13].

Die Fachgesellschaften werden sich daher weiterhin intensiv um eine Qualifikation ihres Berufsstandes in Aus-, Weiter- und Fortbildung kümmern, denn „wer etwas richtig gelernt hat, wird es auch richtig machen“.

\section{Literatur}

1 Aus Fehlern lernen - Aktionsbündnis Patientensicherheit. Bonn: KomPart-Verlagsgesellschaft mbH \& Co KG, 2008

2 Merten M. Fehlerbekenntnis löst Flut an Reaktionen aus. Deutsches Ärzteblatt 2008; 105: B477-B448

3 Merten M. Ungewollte Verunsicherung. Deutsches Ärzteblatt 2005; 102: A1021

4 Scheppokat KD, Neu J. Medizinische Daten und Qualitätsmanagement. Deutsches Ärzteblatt 2007; 104: A3172 - A3177

5 Niethard FU. Wie wichtig ist die Orthopädie für die Studenten? Z Orthop 1999; 137: 199-200

6 Niethard FU. DRG's: Die Orthopädie zwischen Winkelmesser und „Case Mix“. Z Orthop 2001; 139: 277-278

7 Niethard FU. Weiterbildung und Arbeitszeitgesetz: Wie werden aus Ärzten gute Orthopäden? Z Orthop 2001; 139: 467-468

8 Schmidt C, Ramsauer B, Witzel K. Risikomanagement zur Fehlervermeidung im Krankenhaus: Standard Operating Procedures aus der Luftfahrt als Vorbild für eine strukturierte Kommunikation im Klinikalltag. Z Orthop Unfall 2008; 146: 175 - 178

9 Rabbata S. Die Angst steht mit am Operationstisch. Deutsches Ärzteblatt 2003; 100: A668 - A670

10 Hibbeler B. Wenn Sparen zum Risiko wird. Deutsches Ärzteblatt 2007; 104: A2241 - A2242

11 Merten M. Fehlermeldesysteme - Schweiz als Vorreiter. Deutsches Ärzteblatt 2004; 101: A162

12 Weißbuch Schwerverletztenversorgung. Berlin: Deutsche Gesellschaft für Unfallchirurgie e. V., 2006

13 Wienke A. Deutsches Ärzteblatt 2008; 105: A492 